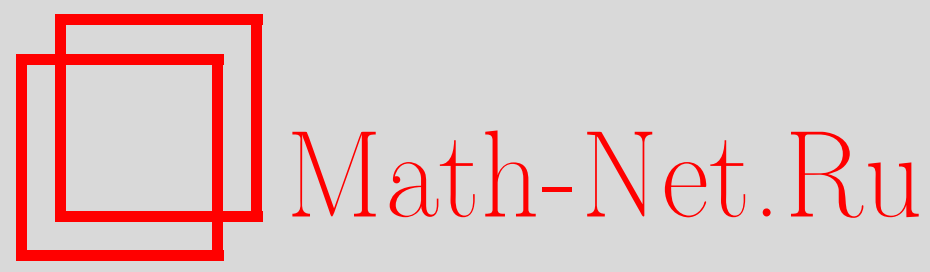

О. Н. Герман, Асимптотические направления для наилучших приближений $n$-мерной линейной формы, Матем. заметки, 2004, том 75, выпуск 1, 55-70

DOI: https://doi.org/10.4213/mzm8

Использование Общероссийского математического портала Math-Net.Ru подразумевает, что вы прочитали и согласны с пользовательским соглашением http://www.mathnet.ru/rus/agreement

Параметры загрузки:

IP : 34.229 .108 .108

26 апреля 2023 г., 12:59:08 


\section{АСИМПТОТИЧЕСКИЕ НАПРАВЛЕНИЯ ДЛЯ НАИЛУЧШИХ ПРИБЛИЖЕНИЙ $n$-МЕРНОЙ ЛИНЕЙНОЙ ФОРМЫ}

\section{О.Н. Герман}

Доказаны некоторые факты об асимптотических направлениях для наилучших приближений линейных форм, аналогичные известным результатам для наилучших совместных приближений.

Библиография: 10 названий.

1. Введение. В этой работе мы будем исследовать асимптотические направления для наилучших приближений и односторонних наилучших приближений линейных форм и получим для них результаты, аналогичные результатам К. А. Роджерса (см. [1], [2]) и Н. Г. Мощевитина (см. [3]) для наилучших совместных приближений.

Введем несколько обозначений, которыми мы будем пользоваться на протяжении всей pаботы. Для $x=\left(x_{1}, \ldots, x_{n}\right) \in \mathbb{R}^{n}$ положим $\underline{x}=\left(x_{2}, \ldots, x_{n}\right) \in \mathbb{R}^{n-1}$. Для $x=$ $\left(x_{1}, \ldots, x_{n}\right) \in \mathbb{Z}^{n}$ положим $\mathbb{M}_{n}(x)=\left\{\left(x_{0}, x_{1}, \ldots, x_{n}\right) \in \mathbb{R}^{n+1} \mid x_{0} \in \mathbb{Z}\right\}$. Иначе говоря, $\mathbb{M}_{n}(x)=\left\{X \in \mathbb{Z}^{n+1} \mid \underline{X}=x\right\}$. Для $\alpha \in \mathbb{R}^{n}$ положим

$$
\begin{array}{ll}
\mathscr{L}_{\alpha}: \mathbb{R}^{n} \rightarrow \mathbb{R}, & \mathscr{L}_{\alpha}(x)=\left(\alpha_{1} x_{1}+\cdots+\alpha_{n} x_{n}\right), \\
\overline{\mathscr{L}_{\alpha}}: \mathbb{R}^{n+1} \rightarrow \mathbb{R}, & \overline{\mathscr{L}_{\alpha}}(X)=\left(X_{0}+\alpha_{1} X_{1}+\cdots+\alpha_{n} X_{n}\right) .
\end{array}
$$

Пусть $\|\cdot\|$ - расстояние до ближайшего целого числа, $\{\cdot\}$ - дробная часть числа, $|\cdot|$ - произвольная фиксированная норма в $\mathbb{R}^{n}$, задаваемая выпуклой симметричной лучевой функцией $f(x), B_{f}^{\delta}(\alpha)=\left\{x \in \mathbb{R}^{n}|| x-\alpha \mid \leqslant \delta\right\}, S_{f}=\partial B_{f}^{1}(0), S_{f}^{*}=\{x=$ $\left(x_{1}, \ldots, x_{n}\right) \in S_{f} \mid x_{1}, \ldots, x_{n}$ линейно независимы над $\left.\mathbb{Z}\right\}$. Ясно, что $S_{f}^{*}$ всюду плотно в $S_{f}$.

Определим функцию натурального аргумента $\Delta(n)$ следующим образом. Положим $\Delta(1)$ равным произвольному фиксированному числу, меньшему $29 / 22$. При $n \geqslant 2$ положим $\Delta(n)=1$.

Мы будем считать, что норма | | такова, что в единичном шаре $B_{f}^{1}(0)$ есть ненулевые целые точки, причем все они лежат на границе этого шара. Это требование не ограничивает общности, однако оно помогает избежать лишних нагромождений при определении некоторых констант.

\section{2. Наилучшие приближения и односторонние наилучшие приближения линейных форм.}

Работа выполнена при финансовой поддержке Российского фонда фундаментальных исследований, грант № 02-01-00912, и INTAS. 
ОПРЕДЕЛЕНИЕ 1. Если $\alpha \in \mathbb{R}^{n}, m \in \mathbb{Z}^{n}$ и $\left\|\mathscr{L}_{\alpha}(m)\right\|<\left\|\mathscr{L}_{\alpha}\left(m^{\prime}\right)\right\|$ для любого $m^{\prime} \in$ $\mathbb{Z}^{n} \backslash\{0, \pm m\}$ такого, что $\left|m^{\prime}\right| \leqslant|m|$, то $m$ будем называть $f$-наилучиим приближением (сокращенно $f$-НП) линейной формы $\mathscr{L}_{\alpha}$.

ОПРеДЕЛЕНИЕ 2. Если $\alpha \in \mathbb{R}^{n}, m \in \mathbb{Z}^{n}$ и $\left\{\mathscr{L}_{\alpha}(m)\right\}<\left\{\mathscr{L}_{\alpha}\left(m^{\prime}\right)\right\}$ для любого $m^{\prime} \in$ $\mathbb{Z}^{n} \backslash\{0, m\}$ такого, что $\left|m^{\prime}\right| \leqslant|m|$, то $m$ будем называть односторонним $f$-наилучиим приближением (сокращенно $f$-ОНП) линейной формы $\mathscr{L}_{\alpha}$.

Определение 1 является классическим. Определение 2 уже в одномерном случае отличается от классического определения (последнее см., например, в [4]).

Из определения 1 видно, что если $m$ является $f$-НП линейной формы $\mathscr{L}_{\alpha}$, то и $-m$ также является $f$-НП $\mathscr{L}_{\alpha}$. То есть множество всех $f$-НП $\mathscr{L}_{\alpha}$ разбивается на пары точек. Более того, ясно, что если $m$ - такое $f$-НП $\mathscr{L}_{\alpha}$, что $0<\left\{\mathscr{L}_{\alpha}(m)\right\}<1 / 2$, то $m$ является еще и $f$-ОНП $\mathscr{L}_{\alpha}$. В обратную сторону: если $m-f$-ОНП $\mathscr{L}_{\alpha}$, то $m-f$-НП $\mathscr{L}_{\alpha}$. Таким образом, множество всех $f$-ОНП $\mathscr{L}_{\alpha}$ - это "половина" множества всех $f$-НП $\mathscr{L}_{\alpha}$.

В работе мы будем исследовать линейные формы только для тех $\alpha=\left(\alpha_{1}, \ldots, \alpha_{n}\right) \in$ $\mathbb{R}^{n}$, для которых $\alpha_{1}, \ldots, \alpha_{n}$ линейно независимы вместе с единицей над $\mathbb{Z}$. В этом (и только в этом) случае множество всех $f$-НП $\mathscr{L}_{\alpha}$ бесконечно.

Упорядочим все $f$-ОНП линейной формы $\mathscr{L}_{\alpha}$ по возрастанию нормы $|m|$. Получим последовательность $\left\{m_{i}\right\}_{i=1}^{\infty}$. Ясно, что множество $\left\{ \pm m_{i}\right\}_{i=1}^{\infty}-$ это множество всех $f$-НП $\mathscr{L}_{\alpha}$.

Если для произвольной ненулевой точки $m$ соответствующим направлением $m^{*}$ в норме $|\cdot|$ назвать точку $m^{*}=m /|m| \in S_{f}$, то последовательности $\left\{m_{i}\right\}_{i=1}^{\infty} f$-ОНП $\mathscr{L}_{\alpha}$ будет однозначно соответствовать последовательность $\left\{m_{i}^{*}\right\}_{i=1}^{\infty}$ направлений $f$-ОНП $\mathscr{L}_{\alpha}$ (все точки этой последовательности различны).

На направления последовательных наилучших приближений не имеется никаких ограничений. Однако, направления последовательных односторонних наилучших приближений не могут находиться слишком близко друг к другу. Для этих направлений имеет место факт, аналогичный теореме о направлениях отклонений наилучших совместных приближений из [3].

ТЕОрема 1. Пусть $\left\{m_{i}\right\}_{i=1}^{\infty}-$ последовательные $f$-ОНП $\mathscr{L}_{\alpha}, a\left\{m_{i}^{*}\right\}_{i=1}^{\infty}-$ направления последовательных $f$-ОНП $\mathscr{L}_{\alpha}$. Тогда

$$
m_{i} \notin \operatorname{int} B_{f}^{\left|m_{i+1}\right|}\left(m_{i+1}\right), \quad m_{i}^{*} \notin \operatorname{int} B_{f}^{1}\left(m_{i+1}^{*}\right) .
$$

ДокАЗАТЕЛЬСТво. По определению $f$-ОНП в слое

$$
H_{i}=\left\{x=\left(x_{0}, x_{1}, \ldots, x_{n}\right) \in \mathbb{R}^{n+1}|| \underline{x}|<| m_{i+1}|,| \overline{\mathscr{L}_{\alpha}}(x) \mid<\left\{\mathscr{L}_{\alpha}\left(m_{i}\right)\right\}\right\}
$$

ненулевых целых точек нет.

Имеет место неравенство $\left\{\mathscr{L}_{\alpha}\left(m_{i+1}\right)\right\}<\left\{\mathscr{L}_{\alpha}\left(m_{i}\right)\right\}$, откуда получаем, что

$$
\left\{\mathscr{L}_{\alpha}\left(m_{i}-m_{i+1}\right)\right\}<\left\{\mathscr{L}_{\alpha}\left(m_{i}\right)\right\}
$$

Допустим теперь, что $m_{i} \in \operatorname{int} B_{f}^{\left|m_{i+1}\right|}\left(m_{i+1}\right)$, т.е. что

$$
\left|m_{i}-m_{i+1}\right|<\left|m_{i+1}\right|
$$


Тогда получим, что существует ненулевая целая точка из $\mathbb{M}_{n}\left(m_{i}-m_{i+1}\right)$, которая попадает в $H_{i}$. Пришли к противоречию. Стало быть,

$$
m_{i} \notin \operatorname{int} B_{f}^{\left|m_{i+1}\right|}\left(m_{i+1}\right) .
$$

Из этого соотношения следует, что

$$
m_{i}^{*} \frac{\left|m_{i}\right|}{\left|m_{i+1}\right|}=\frac{m_{i}}{\left|m_{i+1}\right|} \notin \operatorname{int} B_{f}^{1}\left(m_{i+1}^{*}\right) .
$$

Поскольку $0 \in \partial B_{f}^{1}\left(m_{i+1}^{*}\right)$, a $m_{i}^{*} \frac{\left|m_{i}\right|}{\left|m_{i+1}\right|}-$ внутренняя точка отрезка $\left[0, m_{i}^{*}\right]$, в силу выпуклости $B_{f}^{1}\left(m_{i+1}^{*}\right)$ имеем

$$
m_{i}^{*} \notin \operatorname{int} B_{f}^{1}\left(m_{i+1}^{*}\right)
$$

Заметим, что если норма $|\cdot|$ строго вьпукла, то $m_{i}^{*} \notin B_{f}^{1}\left(m_{i+1}^{*}\right)$, так как иначе $m_{i}^{*} \frac{\left|m_{i}\right|}{\left|m_{i+1}\right|}$ будет внутренней точкой шара $B_{f}^{1}\left(m_{i+1}^{*}\right)$.

\section{3. Асимптотические направления.}

ОПРЕДЕЛЕНИЕ 3 . Пусть $\left\{m_{i}\right\}_{i=1}^{\infty}-$ множество всех $f$-ОНП $\mathscr{L}_{\alpha}$. Тогда $\omega \in S_{f}$ будем назьвать асимптотическим направлением для $f$-ОНП линейной формы $\mathscr{L}_{\alpha}$, если $\omega$ является предельной точкой последовательности $\left\{m_{i}^{*}\right\}_{i=1}^{\infty}$. Соответственно, $\omega$ будем назьвать асимптотическим направлением для $f$-НП линейной формы $\mathscr{L}_{\alpha}$, если $\omega$ является предельной точкой множества $\left\{ \pm m_{i}^{*}\right\}_{i=1}^{\infty}$.

Очевидно, что для любого $\alpha \in \mathbb{R}^{n}$ множество всех асимптотических направлений для $f$-ОНП $\mathscr{L}_{\alpha}$ замкнуто. Однако в силу теоремы 1 не для всякого компакта $\Omega$ на сфере $S_{f}$ найдется такое $\alpha \in \mathbb{R}^{n}$, что множество всех асимптотических направлений для $f$-ОНП $\mathscr{L}_{\alpha}$ совпадает с $\Omega$. Например, $\Omega$ не может иметь диаметр, меньший 1 , так как тогда, начиная с некоторого момента, никакие два элемента последовательности $\left\{m_{i}^{*}\right\}_{i=1}^{\infty}$ не будут удовлетворять соотношению из теоремы 1 . Тем не менее, наложив на множество $\Omega$ некоторые ограничения, мы докажем аналог теоремы 6 из [3] для наилучших совместных приближений. Для этого нам понадобится понятие освещаемых точек. Вообще говоря, существует несколько определений освещаемости. Мы будем пользоваться определением из [5].

ОПРЕДЕЛЕНИЕ 4. Пусть $M$ - замкнутое выпуклое множество, $b \in \partial M, a \notin M$. Тогда будем говорить, что $M$ освещается точкой а в точке $b$, если существует такое $\lambda>0$, что $b+\lambda(b-a)$ содержится в int $M$.

ОПРЕДЕЛЕНИЕ 5. Множество $\Omega \subseteq S_{f}$ будем назьвать асимптотически допустимы., если на сфере $S_{f}$ найдется последовательность $\left\{\theta_{k}\right\}_{k=1}^{\infty}$ такая, что

1) для любого $k$ множество $B_{f}^{1}\left(\theta_{k+1}\right)$ освешается точкой $\theta_{k}$ в точке начала координат;

2) $\Omega$ совпадает с множеством всех предельных точек последовательности $\left\{\theta_{k}\right\}$.

Из второго пункта этого определения видно, что всякое асимптотически допустимое множество непусто и замкнуто. Ясно, что асимптотически допустимые множества существуют. Более того, множество асимптотически допустимых множеств весьма велико. Например, любое замкнутое множество на сфере $S_{f}$, в котором есть хотя бы одна пара центрально симметричных точек, является асимптотически допустимым (см. следствие из леммы 3). 
ТЕОрема 2. Пусть $\Omega$ - асимптотически допустимое множество на сфере $S_{f}$. Тогда существует континуальный набор векторов $\alpha$ из $\mathbb{R}^{n}$ такой, что для любого $\alpha$ из этого набора мноэсество всех асимптотических направлений для f-ОНП $\mathscr{L}_{\alpha}$ coвnaдaem c $\Omega$.

Заметим, что теорему 6 из [3] можно сформулировать в более общей форме, если воспользоваться определением 5 асимптотически допустимых множеств в терминах освещаемых точек.

Теорему 2 мы получим как следствие следующей теоремы. Она составляет основной результат работы.

ТЕОРемА 3. Пусть $\left\{\omega_{i}\right\}_{i=1}^{\infty} \subset S_{f}^{*}-$ такая последовательность, что для любого $i$ множсество $B_{f}^{1}\left(\omega_{i+1}\right)$ освещ,ается точкой $\omega_{i}$ в точке начала координат. Пусть $\left\{\varepsilon_{i}\right\}_{i=1}^{\infty} \subset \mathbb{R}_{+}, \quad \varepsilon_{i}$ убивает $\kappa 0$ сколь угодно быстро при $i \rightarrow \infty$. Пусть к тому же существует челая точка $m \in S_{f}$, освещающая мнохество $B_{f}^{1}\left(\omega_{2}\right)$ в точке начала координат, такая, что $\left|\omega_{1}-m\right| \leqslant \varepsilon_{1}$. Тогда существует такое $\alpha \in \mathbb{R}^{n}$, что для последовательности $\left\{m_{i}\right\}_{i=1}^{\infty}$ всех $f$-ОНП $\mathscr{L}_{\alpha}$ выполняется

$$
\left|\omega_{i}-\frac{m_{i}}{\left|m_{i}\right|}\right| \leqslant \varepsilon_{i}\left|m_{i}\right|^{-\Delta(n-1)} \quad \forall i \in \mathbb{N} \text {. }
$$

Отметим, что в доказательстве теоремы 3, наряду с техникой, напоминающей технику работы [6], используются диофантовы неравенства с простыми числами, недавно полученные Г. Харманом [7], [8]. Отметим также, что более слабьй результат (для $\Delta(n) \equiv 0)$ можно получить без использования сложных результатов о разрешимости диофантовых неравенств с простьми числами.

Рассмотрим теперь $f$-НП $\mathscr{L}_{\alpha}$. Из определения 3 видно, что для любого $\alpha \in \mathbb{R}^{n}$ множество всех асимптотических направлений для $f$-НП $\mathscr{L}_{\alpha}$ замкнуто и центрально симметрично. Из теоремы 2 следует обращение этого факта.

ТЕОРемА 4. Пусть $\Omega$-иентрально симметричное замкнутое множество на сфере $S_{f}$. Тогда существует континуальный набор векторов $\alpha$ из $\mathbb{R}^{n}$ такой, что для любого $\alpha$ из әтого набора множество всех асимптотических направлений для $f$-НП $\mathscr{L}_{\alpha}$ cовпадает $c \Omega$.

Действительно, любое центрально симметричное множество на сфере $S_{f}$ является асимптотически допустимым. Но если $\Omega$ - множество всех асимптотических направлений для $f$-ОНП $\mathscr{L}_{\alpha}$, то $\Omega \cup(-\Omega)$ - множество всех асимптотических направлений для $f-\mathrm{H} \Pi \mathscr{L}_{\alpha}$.

ЗАмЕчАниЕ 1. Из определения асимптотических направлений видно, что множество всех асимптотических направлений для $f$-ОНП $\mathscr{L}_{\alpha}$ пусто тогда и только тогда, когда $\alpha_{1}, \ldots, \alpha_{n}$ линейно зависимы вместе с единицей над $\mathbb{Z}$. Поэтому теоремы 2 и 4 верны и для пустых множеств.

ЗАмечАниЕ 2. К. А. Роджерс в своей работе [1] рассматривает совместные приближения порядка $O(f(x))$ для $f(x)=o(1)$ и порядка $o(f(x))$ для $f(x)=O(1)$. Им доказано, что для произвольной функции $f(x)$ порядка $o(1)$ и произвольного непустого замкнутого подмножества $\Omega$ единичной сферы, симметричного относительно начала координат, найдется вектор, для которого множества асимптотических направлений порядка $O(f(x))$ и порядка $o(f(x))$ совпадают с $\Omega$, что весьма напоминает теорему 4. 


\section{4. Некоторые свойства освещаемости.}

Лемма 1. Мнохсество точек, освещающих $M$ в точке $b$, является откритым конусом с верииной в точке $b$.

Лемма 1 очевидна, и мы ее не доказываем.

Лемма 2. Множество точек $x \in \mathbb{R}^{n}$ таких, что точка а освещает иар $B_{f}^{|x-b|}(x)$ в точке $b$, является открытым конусом с вершиной в точке $b$.

ДокАЗАТЕльство. Пусть точка $a$ освешает $B_{f}^{|x-b|}(x)$ в точке $b$. Тогда по определению существует такое $\lambda>0$, что $b+\lambda(b-a)$ содержится вместе с некоторой своей окрестностью в $B_{f}^{|x-b|}(x)$. Стало быть, существует такая окрестность точки $x$, что для любого $y$ из этой окрестности шар $B_{f}^{|y-b|}(y)$ содержит точку $b+\lambda(b-a)$. Открытость доказана.

Пусть $\mu>0$. Тогда если

$$
b+\lambda(b-a) \in \operatorname{int} B_{f}^{|x-b|}(x),
$$

то, очевидно,

$$
b+\lambda \mu(b-a) \in \operatorname{int} B_{f}^{|\mu(x-b)|}(b+\mu(x-b)),
$$

что доказьвает лемму.

Лемма 3. Пусть $a, b \in S_{f}$ u nусть $[0, a] \cap \operatorname{int} B_{f}^{1}(b)=\varnothing$. Тогда для любого $\varepsilon>0$ найдутся такие точки $a^{\prime}, b^{\prime} \in S_{f}$, что $\left|a-a^{\prime}\right|<\varepsilon,\left|b-b^{\prime}\right|<\varepsilon$ и а о освещает $B_{f}^{1}\left(b^{\prime}\right)$ в точке начала координат.

ДокАЗАТЕЛЬСтво. Поскольку $0 \in \operatorname{int} B_{f}^{1}(0)$, а множество $B_{f}^{1}(0)$ выпукло, то внутренность 2 -мерного (или 1-мерного) множества $\langle a, b\rangle_{\mathbb{R}} \cap B_{f}^{1}(b)$ содержится в int $B_{f}^{1}(b)$. Следовательно, достаточно доказать лемму в размерности $n=2$.

Если $n=2$, то прямая $\langle b\rangle_{\mathbb{R}}$ делит все пространство на две полуплоскости, левую и правую. Пусть точка $a$ лежит в правой полуплоскости. Тогда при достаточно малом $\varepsilon>0$ для любой точки $b^{\prime} \in S_{f}$ из левой $\varepsilon$-окрестности точки $b$ выполняется $[0, a] \cap$ int $B_{f}^{1}\left(b^{\prime}\right)=\varnothing$. Далее, из теоремы Лебега о дифференцируемости монотонной функции почти всюду следует, что вьпуклая на отрезке функция также почти всюду дифференцируема. Следовательно, в левой $\varepsilon$-окрестности точки $b$ можно найти точку $b^{\prime}$, в которой существует касательная к шару $B_{f}^{1}(0)$. В $\varepsilon$-окрестности $a$ можно найти точку $a^{\prime}$, не лежащую на этой касательной и для которой тоже вьполняется $\left[0, a^{\prime}\right] \cap \operatorname{int} B_{f}^{1}\left(b^{\prime}\right)=\varnothing$. Но тогда для некоторого $\lambda>0-\lambda a^{\prime} \in \operatorname{int} B_{f}^{1}\left(b^{\prime}\right)$, т.е. $a^{\prime}$ освещает $B_{f}^{1}\left(b^{\prime}\right)$ в точке начала координат.

СЛЕДСТВИЕ. Пусть $\Omega \subset S_{f}-$ замкнутое мнохество, в котором есть хотя бъ одна пара иентрально-симметричных точек $\pm \omega$. Тогда $\Omega$ асимптотически допустимо.

ДокаЗАтЕльСтво. Рассмотрим пространство $\Theta$ последовательностей $\left\{\theta_{k}\right\}_{k=1}^{\infty} \subset$ $S_{f}$ и определим отображение $\varphi_{\omega}: \Theta \rightarrow \Theta$ следующим образом. Пусть $\theta \in \Theta$ и пусть $k_{o}-$ минимальное $k$ такое, что $\theta_{k}$ не освещает $B_{f}^{1}\left(\theta_{k+1}\right)$ в точке начала координат. Из вьпуклости нормы $|\cdot|$ очевидньм образом следует, что найдутся такие точки $\tau_{1}, \tau_{2} \in\{ \pm \omega\}$, что $\left[0, \theta_{k_{o}}\right] \cap \operatorname{int} B_{f}^{1}\left(\tau_{1}\right)=\varnothing$ и $\left[0, \tau_{2}\right] \cap \operatorname{int} B_{f}^{1}\left(\theta_{k_{o}+1}\right)=\varnothing$. По лемме 3 найдутся такие 
точки $\theta_{k_{o}}^{\prime}, \tau_{1}^{\prime} \in S_{f}$, что $\left|\theta_{k_{o}}-\theta_{k_{o}}^{\prime}\right|<2^{-k_{o}},\left|\tau_{1}-\tau_{1}^{\prime}\right|<2^{-k_{o}}$ и $\theta_{k_{o}}^{\prime}$ освещает $B_{f}^{1}\left(\tau_{1}^{\prime}\right)$ в точке начала координат. В силу лемм 1,2 точки $\theta_{k_{o}}^{\prime}, \tau_{1}^{\prime}$ можно выбрать таким образом, чтобы $\theta_{k_{o}-1}$ освешала шар $B_{f}^{1}\left(\theta_{k_{o}}^{\prime}\right)$, а $\tau_{1}^{\prime}$ - шар $B_{f}^{1}\left(-\tau_{1}\right)$ в точке начала координат.

Возможны два случая: $\tau_{1}=\tau_{2}$ и $\tau_{1}=-\tau_{2}$. Для удобства положим $\tau^{\prime \prime}=-\tau_{1}$, если $\tau_{1}=\tau_{2}$ и $\tau^{\prime \prime}=\tau_{1}^{\prime}$, если $\tau_{1}=-\tau_{2}$. Применим опять лемму 3 , на этот раз к точкам $\tau_{2}$, $\theta_{k_{o}+1}$. Получим точки $\tau_{2}^{\prime}, \theta_{k_{o}+1}^{\prime} \in S_{f}$ такие, что $\left|\tau_{2}-\tau_{2}^{\prime}\right|<2^{-k_{o}},\left|\theta_{k_{o}+1}-\theta_{k_{o}+1}^{\prime}\right|<2^{-k_{o}}$ и $\tau_{2}^{\prime}$ освещает $B_{f}^{1}\left(\theta_{k_{o}+1}^{\prime}\right)$ в точке начала координат. И вновь в силу леммы 2 точку $\tau_{2}^{\prime}$ можно выбрать таким образом, чтобы $\tau^{\prime \prime}$ освешала шар $B_{f}^{1}\left(\tau_{2}^{\prime}\right)$ в точке начала координат.

Перейдем непосредственно к определению $\varphi_{\omega}(\theta)$. Если $\tau_{1}=\tau_{2}$, то заменим фрагмент $\left\{\theta_{k_{o}}, \theta_{k_{o}+1}\right\}$ последовательности $\theta$ на $\left\{\theta_{k_{o}}^{\prime}, \tau_{1}^{\prime},-\tau_{1}, \tau_{2}^{\prime}, \theta_{k_{o}+1}^{\prime}\right\}$, а если $\tau_{1}=-\tau_{2},-$ то на $\left\{\theta_{k_{o}}^{\prime}, \tau_{1}^{\prime}, \tau_{2}^{\prime}, \theta_{k_{o}+1}^{\prime}\right\}$. Получившуюся последовательность и возьмем в качестве $\varphi_{\omega}(\theta)$.

Рассмотрим теперь последовательность $\theta=\left\{\theta_{k}\right\}_{k=1}^{\infty} \subseteq \Omega$, всюду плотную в $\Omega$, и положим

$$
\theta^{\prime}=\lim _{m \rightarrow \infty} \varphi_{\omega}^{m}(\theta)
$$

Нетрудно видеть, что последовательность $\theta^{\prime}$ удовлетворяет всем требованиям определения 5. Следовательно, множество $\Omega$ асимптотически допустимо.

5. Аналог теоремы о сигнатуре для односторонних наилучших приближений линейных форм. Сигнатурой вектора $\omega=\left(\omega_{1}, \ldots, \omega_{n}\right)$ назьвается набор $\operatorname{sign} \omega=\left(\operatorname{sign} \omega_{1}, \ldots, \operatorname{sign} \omega_{n}\right)$. Для обычных ОНП $\mathscr{L}_{\alpha}$, т.е. в случае, когда $|x|=$ $\max \left(\left|x_{1}\right|, \ldots,\left|x_{n}\right|\right)$, из теоремы 1 следует, что $\operatorname{sign} m_{k+1} \neq \operatorname{sign} m_{k}$. Для наилучших совместных приближений аналогичный факт был доказан Роджерсом [2] и впоследствии был обобщен Мощевитиным [3].

Для наилучших совместных приближений в [9] показано, что для любой последовательности сигнатур $\left\{\sigma_{i}\right\}_{i=1}^{\infty}$ такой, что $\sigma_{i} \neq \sigma_{i+1}$, существует набор $\alpha \in \mathbb{R}^{n}$ чисел $\left(\alpha_{1}, \ldots, \alpha_{n}\right)$, независимых вместе с 1 над $\mathbb{Z}$, таких, что $\operatorname{sign} \xi_{i}=\sigma_{i}$, где $\xi_{i}-$ отклонение от $i$-го наилучшего приближения вектора $\alpha$.

Докажем аналогичный факт для обычных ОНП $\mathscr{L}_{\alpha}$. Пусть далее в этом разделе $|x|=$ $\max \left(\left|x_{1}\right|, \ldots,\left|x_{n}\right|\right)$.

ТЕОрема 5. Если задана последовательность сигнатур $\left\{\sigma_{i}\right\}_{i=1}^{\infty}$ такая, что $\sigma_{i} \neq \sigma_{i+1}$ и все координаты этих сигнатур отличны от нуля, то существует вектор $\alpha \in \mathbb{R}^{n}$ такой, что $\operatorname{sign} m_{i}=\sigma_{i}$ для всех $i$, әде $m_{i}-i$-е ОНП $\mathscr{L}_{\alpha}$.

Для доказательства теоремы 5 построим индукцией по $i$ последовательность $\left\{\omega_{i}\right\}_{i=1}^{\infty} \subset S_{f}^{*}$ такую, что $\operatorname{sign} \omega_{i}=\sigma_{i}$ и $\omega_{i}$ освешает $B_{f}^{1}\left(\omega_{i+1}\right)$ в точке начала координат. Для сигнатуры $\sigma$ положим

$$
U(\sigma)=\left\{x \in S_{f}^{*} \mid \operatorname{sign} x=\sigma\right\}
$$

Лемма. Пусть $\sigma, \sigma^{\prime}-$ различные сигнатуры без нулевых координат. Тогда в $U\left(\sigma^{\prime}\right)$ найдется такая точка $\omega^{\prime}$, что любая точка $\omega \in U(\sigma)$ освещает множество $B_{f}^{1}\left(\omega^{\prime}\right)$ в точке начала координат.

ДокАЗАтЕЛЬСтво. Поскольку $\sigma \neq \sigma^{\prime}$, существует такое число $j \in\{1, \ldots, n\}$, что $U(\sigma)$ и $U\left(\sigma^{\prime}\right)$ лежат по разные стороны от координатной гиперплоскости $\pi=\left\{x \in \mathbb{R}^{n} \mid\right.$ $\left.x_{j}=0\right\}$.

В качестве $\omega^{\prime}$ возьмем любую точку из множества $\left\{x \in U\left(\sigma^{\prime}\right)|| x_{j}|=1,| x_{i} \mid \neq 1\right.$, $i \neq j\}$. Тогда множество $U(\sigma)$ и шар $B_{f}^{1}\left(\omega^{\prime}\right)$ находятся по разные стороны от плоскости 
$\pi$, причем начало координат лежит во внутренности $(n-1)$-мерной грани шара $B_{f}^{1}\left(\omega^{\prime}\right)$, откуда и следует утверждение леммы.

Эта лемма доказывает шаг индукции. Основание индукции очевидно. В итоге получаем последовательность $\left\{\omega_{i}\right\}_{i=1}^{\infty} \subset S_{f}^{*}$ такую, что $\operatorname{sign} \omega_{i}=\sigma_{i}$ и $\omega_{i}$ освещает $B_{f}^{1}\left(\omega_{i+1}\right)$ в точке начала координат при любом $i$. Возьмем теперь подходящую последовательность $\left\{\varepsilon_{i}\right\}_{i=1}^{\infty}$ и применим теорему 3 . Получим требуемую линейную форму.

Заметим, что из теоремы 4 работы [3] следует аналогичньй результат для наилучших совместных приближений, полученный ранее в [9]. Эту теорему можно сформулировать в более общей форме, если воспользоваться понятием освещаемости.

ТЕОрема 6. Пусть последовательность $\left\{\theta_{i}\right\}_{i=1}^{\infty} \subset S_{f}$ такова, что для любого $i$ мнохсество $B_{f}^{1}\left(\theta_{i}\right)$ освещается точкой $\theta_{i+1}$ в точке начала координат. Тогда существует набор $\alpha=\left(\alpha_{1}, \ldots, \alpha_{n}\right)$ чисел, линейно независимых вместе с единиией над $\mathbb{Z}$, такой, что

$$
\lim _{i \rightarrow \infty}\left|\theta_{i}-\frac{\xi_{i}}{\left|\xi_{i}\right|}\right|=0,
$$

əде $\left\{\xi_{i}\right\}_{i=1}^{\infty}$ - последовательность отклонений от всех наилучиих совместных приближений вектора $\alpha$.

Не удивительно, что для совместных приближений $\alpha$ в теореме 6 мы требуем "обратную” освещаемость, в отличие от теоремы 3 для ОНП $\mathscr{L}_{\alpha}$. Такое же явление наблюдается и в результатах типа теоремы 1.

Как было сказано выше, из теоремы 6 следует

ТЕОРема 7. Если задана последовательность сигнатур $\left\{\sigma_{i}\right\}_{i=1}^{\infty}$ такая, что $\sigma_{i} \neq \sigma_{i+1}$ и все координаты этих сигнатур отличны от нуля, то существует вектор $\alpha \in \mathbb{R}^{n}$ такой, что $\operatorname{sign} \xi_{i}=\sigma_{i}$ для всех $i$, әде $\xi_{i}$ - отклонение от $i$-го наилучшего приближения вектора $\alpha$.

Вывод теоремы 7 из теоремы 6 аналогичен выводу теоремы 5 из теоремы 3. Единственньм существенным отличием является то, что нужно строить последовательность $\left\{\theta_{i}\right\}_{i=1}^{\infty}$ такую, что $\operatorname{sign} \theta_{i}=\sigma_{i}$ и $\theta_{i+1}$ освешает $B_{f}^{1}\left(\theta_{i}\right)$ в точке начала координат. Если уже выбраны $\theta_{i}, i \leqslant k$, то в зависимости от того, каково $\sigma_{k+2}$, выбираем $\theta_{k+1} \in$ $U\left(\sigma_{k+1}\right)$, пользуясь все той же леммой.

Хотелось бы сделать еще одно замечание по поводу теоремы 6. В тексте доказательства этой теоремы в [3] имеется недочет, который легко устранить, если на $\nu$-м шаге индукции требовать вдобавок ко всему, чтобы для всех $k=1,2, \ldots, \nu-2$ на боковых поверхностях цилиндров

$$
\Omega_{k}=\left\{z=\left(x, y_{1}, \ldots, y_{n}\right) \in \mathbb{R}^{n+1}|| x \mid<p_{k+1}, f(\alpha x-y)<f\left(\xi_{k}\right)\right\}
$$

не было целых точек, кроме наилучших приближений.

\section{6. Вспомогательные утверждения.}

Лемма 4. Пусть $x^{1}, x^{2}, x^{3} \in \mathbb{R}^{n+1}$. Пусть $\overline{\mathscr{L}_{\alpha}}\left(x^{i}\right)=0$ для всех $\alpha \in \pi \subset \mathbb{R}^{n}$, дде $\pi$-некоторая $(n-2)$-мерная плоскость, $0 \notin \pi$. Тогда $x^{1}, x^{2}, x^{3}$ линейно зависимы над $\mathbb{R}$.

Лемма 4 очевидна и мы ее не доказываем. 
Лемма 5. Пусть вектор $\omega=\left(\omega_{1}, \ldots, \omega_{n}\right) \in \mathbb{R}^{n}$ таков, что $\omega_{1}, \ldots, \omega_{n}$ линейно независимы вместе с 1 над $\mathbb{Z}$. Пусть $\varepsilon>0$. Тогда существует бесконечно много простых $q$ и точек $b \in \mathbb{Z}^{n}$ таких, что

$$
|q \omega-b| \leqslant \varepsilon q^{1-\Delta(n)} .
$$

Если $n=1$, то лемма 5 вытекает из следующей теоремы, доказанной $\Gamma$. Харманом [7], которая является усилением известной теоремы Виноградова [10].

ТеОремА. Пусть а ирраиионально. Тогда для всех действительных $\beta$ существует бесконечно много простых q таких, что

$$
\|\alpha q+\beta\|<q^{-7 / 22} .
$$

Если же $n \geqslant 2$, то лемма 5 следует из того факта, что

$$
\liminf _{q \rightarrow \infty} \max _{1 \leqslant i \leqslant n}\left\|q \omega_{i}\right\|=0
$$

если $\omega_{1}, \ldots, \omega_{n}$ линейно независимы вместе с 1 над $\mathbb{Z}$, а $q$ простое. Этот факт был доказан Харманом в [8]. В случае $n \geqslant 2$ невозможно получить никакую равномерную по $\omega$ оценку на стремление к нулю $|q \omega-b|$ лучше, чем константа (см. контрпример в [8]).

Лемма 6. Пусть вектор $\omega=\left(\omega_{1}, \ldots, \omega_{n}\right) \in \mathbb{R}^{n}$ таков, что $\omega_{1}, \ldots, \omega_{n}$ линейно независимы вместе с 1 над $\mathbb{Z}$. Пусть $\varepsilon>0$. Тогда существует бесконечно много иельх р и точек $b=\left(b_{1}, \ldots, b_{n}\right) \in \mathbb{Z}^{n}$ таких, что $\left|b_{n}\right|-$ простое число $u$

$$
|p \omega-b| \leqslant \varepsilon p^{1-\Delta(n)} .
$$

ДОКАЗАТЕЛЬСТВО. Положим

$$
\begin{gathered}
\varepsilon_{1}=\frac{\varepsilon}{\left(2\left|\omega_{n}\right|\right)^{1-\Delta(n)} \max \left(1+\left|\omega_{1}\right|, \ldots, 1+\left|\omega_{n-1}\right|,\left|\omega_{n}\right|\right)}, \\
\gamma_{1}=\frac{\omega_{1}}{\omega_{n}}, \quad \ldots, \quad \gamma_{n-1}=\frac{\omega_{n-1}}{\omega_{n}}, \quad \gamma_{n}=\frac{1}{\omega_{n}} .
\end{gathered}
$$

Очевидно, $\gamma_{1}, \ldots, \gamma_{n}$ линейно независимы вместе с 1 над $\mathbb{Z}$; следовательно, по лемме 5 существует бесконечно много простых $q$ и точек $a=\left(a_{1}, \ldots, a_{n}\right) \in \mathbb{Z}^{n}$ таких, что

$$
\left|q \gamma_{i}-a_{i}\right| \leqslant \varepsilon_{1} q^{1-\Delta(n)} \quad \forall i=1, \ldots, n,
$$

откуда получаем, что

$$
\left\{\begin{array}{c}
\left|a_{n} \omega_{i}-a_{i}+\left(q-a_{n} \omega_{n}\right) \omega_{i} \omega_{n}^{-1}\right| \leqslant \varepsilon_{1} q^{1-\Delta(n)} \quad \forall i=1, \ldots, n-1, \\
\left|a_{n} \omega_{n}-q\right| \leqslant \varepsilon_{1}\left|\omega_{n}\right| q^{1-\Delta(n)}
\end{array}\right.
$$

Следовательно,

$$
\left\{\begin{array}{l}
\left|a_{n} \omega_{i}-a_{i}\right| \leqslant \varepsilon_{1}\left(1+\left|\omega_{i}\right|\right) q^{1-\Delta(n)} \quad \forall i=1, \ldots, n-1, \\
\left|a_{n} \omega_{n}-q\right| \leqslant \varepsilon_{1}\left|\omega_{n}\right| q^{1-\Delta(n)} .
\end{array}\right.
$$

Легко видеть, что $q \leqslant\left|a_{n} \omega_{n}\right|+\varepsilon_{1}\left|\omega_{n}\right|$. Без ограничения общности можно считать, что $a_{n} \neq 0$ и $\varepsilon_{1} \leqslant\left|a_{n}\right|$, откуда $q \leqslant 2\left|a_{n} \omega_{n}\right|$.

Положим

$$
p=\left|a_{n}\right|, \quad b_{1}=\operatorname{sign}\left(a_{n}\right) a_{1}, \quad \ldots, \quad b_{n-1}=\operatorname{sign}\left(a_{n}\right) a_{n-1}, \quad b_{n}=\operatorname{sign}\left(a_{n}\right) q .
$$

Тогда

$$
\left|p \omega_{i}-b_{i}\right| \leqslant \varepsilon p^{1-\Delta(n)} \quad \forall i=1, \ldots, n,
$$

что доказьвает лемму. 
Лемма 7. Пусть $\omega \in S_{f}^{*}, \varepsilon>0$. Пусть $A \in \mathbb{Z}^{n+1}-$ примитивная точка. Тогда существует бесконечно много $b \in \mathbb{Z}^{n}$ таких, что

1) $\left|\omega-\frac{b}{|b|}\right| \leqslant \varepsilon|b|^{-\Delta(n-1)}$;

2) для любых двух соседних $B_{1} u B_{2} \in \mathbb{M}_{n}(b)$ (m.e. таких $B_{1} u B_{2}$, которые отличаются друг от друга на вектор стандартного базиса $\mathbb{R}^{n}$ ) хотя би одна из двух пар векторов $\left\{A, B_{1}\right\} u\left\{A, B_{2}\right\}$ дополнима до базиса $\mathbb{Z}^{n+1}$.

ДокАЗАтЕльство. Будем считать, что $A=\left(A_{0}, \ldots, A_{n}\right)$. Проекция $\underline{A}$ точки $A$ (полученная отбрасьванием координаты $\left.A_{0}\right)$ не обязательно является примитивной точкой. Поэтому мы расмотрим $a=\underline{A} / \lambda$, где $\lambda=$ НОД $\left(\left|A_{1}\right|, \ldots,\left|A_{n}\right|\right) ; a-$ примитивная точка решетки $\mathbb{Z}^{n}$; следовательно, в $\mathbb{Z}^{n}$ существует базис $\mathscr{F}=\left\{f_{1}, \ldots, f_{n}\right\}$ такой, что $f_{1}=a$. Пусть $G \in \operatorname{Sl}_{n}(\mathbb{Z}), G\left(\mathscr{E}^{n}\right)=\mathscr{F}$ (здесь $\mathscr{E}^{n}=\left\{e_{1}, \ldots, e_{n}\right\}-$ стандартньй базис $\left.\mathbb{R}^{n}\right)$, т.е. $G$ - автоморфизм $\mathbb{Z}^{n}$, переводящий $e_{1}$ в $a$.

Легко видеть, что

$\exists \varepsilon_{1}>0$ : если $\left|\frac{x}{|x|}-\frac{y}{|y|}\right| \leqslant \varepsilon_{1}|z|^{-\Delta(n-1)}, \quad$ то $\left|\frac{G(x)}{|G(x)|}-\frac{G(y)}{|G(y)|}\right| \leqslant \varepsilon|G(z)|^{-\Delta(n-1)}$.

Пусть $G^{-1}(\omega)_{1}-$ первая координата вектора $G^{-1}(\omega)$ - отлична от нуля. Положим

$$
\omega^{\prime}=\frac{G^{-1}(\omega)}{\left|G^{-1}(\omega)_{1}\right|}=\left(1, \frac{G^{-1}(\omega)_{2}}{\left|G^{-1}(\omega)_{1}\right|}, \ldots, \frac{G^{-1}(\omega)_{n}}{\left|G^{-1}(\omega)_{1}\right|}\right) .
$$

Рассмотрим $\omega^{\prime \prime}=\underline{\omega^{\prime}}-$ проекцию $\omega^{\prime}$ вдоль $e_{1}$. Ясно, что $\omega_{2}^{\prime}, \ldots, \omega_{n}^{\prime}$ линейно независимы вместе с 1 над $\mathbb{Z}$. Значит, можно применить лемму 6 . Тогда получим, что для любого $\varepsilon_{2}>0$ существует бесконечно много целых $p$ и точек $b^{\prime \prime} \in \mathbb{Z}^{n-1}$ таких, что $\left|b_{n}^{\prime \prime}\right|-$ простое число и

$$
\left|\omega^{\prime \prime}-\frac{b^{\prime \prime}}{p}\right| \leqslant \varepsilon_{2} p^{-\Delta(n-1)} .
$$

Для всех таких $b^{\prime \prime}$ существует $b^{\prime}=\left(p, b_{2}^{\prime}, \ldots, b_{n}^{\prime}\right) \in \mathbb{M}_{n-1}\left(b^{\prime \prime}\right) \subset \mathbb{Z}^{n}$ такое, что

$$
\left|\omega^{\prime}-\frac{b^{\prime}}{p}\right| \leqslant \varepsilon_{2} p^{-\Delta(n-1)} \text {. }
$$

Из этого неравенства легко видеть, что при фиксированных $\omega$ и $G$ можно выбрать $\varepsilon_{2}$ столь малым, что для бесконечного числа точек $b^{\prime} \in \mathbb{Z}^{n}$ с простой координатой $b_{n}^{\prime}$ будет выполнено

$$
\left|\frac{G^{-1}(\omega)}{\left|G^{-1}(\omega)\right|}-\frac{b^{\prime}}{\left|b^{\prime}\right|}\right| \leqslant \varepsilon_{1}\left|b^{\prime}\right|^{-\Delta(n-1)} .
$$

Зафиксируем такое $b^{\prime}$ и положим $b=G\left(b^{\prime}\right)$. Точка $b$ такова, что

$$
\left|\omega-\frac{b}{|b|}\right| \leqslant \varepsilon|b|^{-\Delta(n-1)} .
$$

Осталось доказать второй пункт леммы для точки $b$. Пусть $\left|b_{n}^{\prime}\right|=q$. Без ограничения общности можно считать, что $q>\lambda$. НОД $\left(\left|b_{2}^{\prime}\right|, \ldots,\left|b_{n}^{\prime}\right|\right)$ равен либо 1 , либо $q$. Мы рассмотрим эти два случая по очереди. 
1) НОД $\left(\left|b_{2}^{\prime}\right|, \ldots,\left|b_{n}^{\prime}\right|\right)=1 . b^{\prime \prime}-$ примитивная точка; следовательно, пара векторов $\left\{e_{1}, b^{\prime}\right\}$ дополнима до базиса $\mathbb{Z}^{n}$, поскольку $b^{\prime \prime}$ - проекция $b^{\prime}$ вдоль $e_{1}\left(b^{\prime \prime}=\underline{b^{\prime}}\right)$. Стало быть, и пара векторов $\{a, b\}$ дополнима до базиса $\mathbb{Z}^{n}$.

Остается показать, что из дополнимости $\{a, b\}$ до базиса $\mathbb{Z}^{n}$ следует дополнимость $\{A, B\}$ до базиса $\mathbb{Z}^{n+1}$ для $\forall B \in \mathbb{M}_{n}(b)$. Заметим, что для $\forall B \in \mathbb{M}_{n}(b)$ решетка $\langle\underline{A}, b\rangle_{\mathbb{Z}}$ является проекцией решетки $\langle A, B\rangle_{\mathbb{Z}}$ на пространство $\left\langle e_{1}, \ldots, e_{n}\right\rangle_{\mathbb{R}}$ параллельно $e_{0}\left(\left\{e_{0}, e_{1}, \ldots, e_{n}\right\}\right.$ - стандартньй базис $\left.\mathbb{R}^{n+1}\right)$.

Пусть $C=x A+y B \in \mathbb{Z}^{n+1}$. Достаточно доказать, что $x, y \in \mathbb{Z}$. Имеем $\underline{C}=x \underline{A}+$ $y b=x \lambda a+y b \in\langle a, b\rangle_{\mathbb{Z}}$, так как пара $\{a, b\}$ дополнима до базиса $\mathbb{Z}^{n}$. Следовательно, $y \in \mathbb{Z}$. Но тогда $x A=C-y B \in \mathbb{Z}^{n+1}$, в то время, как точка $A$ примитивна. Отсюда получаем, что $x \in \mathbb{Z}$.

Дополнимость $\{A, B\}$ до базиса $\mathbb{Z}^{n+1}$ доказана.

2) НОД $\left(\left|b_{2}^{\prime}\right|, \ldots,\left|b_{n}^{\prime}\right|\right)=q$. В этом пункте будем считать $b^{\prime \prime}$ элементом $\mathbb{R}^{n}$, приписав в начало нулевую координату. Это естественно, поскольку $b^{\prime \prime}=\underline{b^{\prime}}$. Тогда все целые точки, которые находятся в плоскости $\left\langle e_{1}, b^{\prime \prime}\right\rangle_{\mathbb{R}}$, лежат в решетке $\left\langle e_{1}, b^{\prime \prime} / q\right\rangle_{\mathbb{Z}}$. $\mathrm{K}$ тому же решетка $\left\langle e_{1}, b^{\prime \prime}\right\rangle_{\mathbb{Z}}$ является подрешеткой простого индекса решетки $\left\langle e_{1}, b^{\prime \prime} / q\right\rangle_{\mathbb{Z}}$.

Обозначим

$$
\Lambda_{A}=\langle\underline{A}, b\rangle_{\mathbb{Z}}, \quad \Lambda_{a}=\langle a, b\rangle_{\mathbb{Z}}, \quad \Lambda=\left\langle a, \frac{G\left(b^{\prime \prime}\right)}{q}\right\rangle_{\mathbb{Z}} .
$$

Ясно, что $\Lambda_{a}$ - подрешетка простого индекса решетки $\Lambda$ в силу аналогичного свойства для прообразов этих решеток.

Пусть $B_{1}, B_{2}$ - соседние точки из $\mathbb{M}_{n}(b)$, т.е. $B_{1}-B_{2}= \pm e_{0} \quad\left(\left\{e_{0}, e_{1}, \ldots, e_{n}\right\}-\right.$ стандартньй базис $\left.\mathbb{R}^{n+1}\right)$. Через $\Lambda_{i}$ обозначим проекции решеток $\left\langle A, B_{i}\right\rangle_{\mathbb{R}} \cap \mathbb{Z}^{n+1}$ на пространство $\left\langle e_{1}, \ldots, e_{n}\right\rangle_{\mathbb{R}}$ вдоль $e_{0}, i=1,2$.

Обозначим $\Lambda_{i}(a)=\left\langle\Lambda_{i}, a\right\rangle_{\mathbb{Z}} \supset \Lambda_{i}$. Тогда

$$
\Lambda_{a} \subseteq \Lambda_{i}(a) \subseteq \Lambda, \quad i=1,2
$$

следовательно,

$$
\Lambda_{i}(a) \in\left\{\Lambda_{a}, \Lambda\right\}, \quad i=1,2,
$$

поскольку $\Lambda_{a}$ - подрешетка простого индекса решетки $\Lambda$. Покажем, что $\Lambda_{1}(a)$ и $\Lambda_{2}(a)$ одновременно не могут быть равны $\Lambda$. Допустим противное:

$$
\Lambda_{1}(a)=\Lambda_{2}(a)=\Lambda
$$

Возьмем $\alpha$ и $\beta \in \mathbb{R}^{n}$ такие, что

$$
\left\{x \in \mathbb{R}^{n+1} \mid \overline{\mathscr{L}_{\alpha}}(x)=0\right\}=\left\langle A, B_{1}\right\rangle_{\mathbb{R}} \quad \text { и } \quad\left\{x \in \mathbb{R}^{n+1} \mid \overline{\mathscr{L}_{\beta}}(x)=0\right\}=\left\langle A, B_{2}\right\rangle_{\mathbb{R}}
$$

Линейные формы $\mathscr{L}_{\alpha}$ и $\mathscr{L}_{\beta}$ принимают целые значения соответственно на $\Lambda_{1}$ и $\Lambda_{2}$. Кроме того, очевидно, что $\lambda \mathscr{L}_{\alpha}(a)=\lambda \mathscr{L}_{\beta}(a) \in \mathbb{Z}$. Стало быть, на решетке $\Lambda_{1}(a)=\Lambda_{2}(a)=$ $\Lambda$ функции $\lambda \mathscr{L}_{\alpha}$ и $\lambda \mathscr{L}_{\beta}$ принимают целые значения. Рассмотрим точку $G\left(b^{\prime \prime}\right) \in \Lambda$. Эта точка представима в виде $b+\mu a$, где $\mu$-некоторое целое число (поскольку $b^{\prime \prime}=\underline{b^{\prime}}$ ). Из линейности получаем, что

$$
\frac{1}{q} \mathscr{L}_{\alpha}\left(G\left(b^{\prime \prime}\right)\right)-\frac{1}{q} \mathscr{L}_{\beta}\left(G\left(b^{\prime \prime}\right)\right)= \pm \frac{1}{q} .
$$


Но $0<\lambda / q<1 ;$ следовательно,

$$
\lambda \mathscr{L}_{\alpha}\left(\frac{G\left(b^{\prime \prime}\right)}{q}\right)-\lambda \mathscr{L}_{\beta}\left(\frac{G\left(b^{\prime \prime}\right)}{q}\right) \notin \mathbb{Z}
$$

в то время, как точка $G\left(b^{\prime \prime}\right) / q$ содержится в $A$. Пришли к противоречию; стало быть, хотя бы одна из решеток $\Lambda_{i}(a)$ равна $\Lambda_{a}$. Допустим, без ограничения обшности, что это $\Lambda_{1}(a)$. Докажем, что

$$
\Lambda_{1} \cap \Lambda_{a}=\Lambda_{A}
$$

Вложение $\Lambda_{A} \subseteq \Lambda_{1} \cap \Lambda_{a}$ сразу следует из определения решеток. Пусть $x \in \Lambda_{1} \cap \Lambda_{a}$. Тогда, во-первых, $x$ представим в виде $x_{1} a+x_{2} b$ с целыми $x_{1}$ и $x_{2}$, во-вторых, $x=\underline{X}$, где $X$ принадлежит решетке $\left\langle A, B_{1}\right\rangle_{\mathbb{R}} \cap \mathbb{Z}^{n+1}$, т.е. $X=\left(x_{1} / \lambda\right) A+x_{2} B_{1} \in \mathbb{Z}^{n+1}$. Но $x_{2}$ целое, значит, $\left(x_{1} / \lambda\right) A \in \mathbb{Z}^{n+1}$. Следовательно, в силу примитивности $A x_{1} / \lambda$ целое, т.е. $x=\underline{X} \in \Lambda_{A}$.

Получаем цепочку равенств: $\Lambda_{1}=\Lambda_{1} \cap \Lambda_{1}(a)=\Lambda_{1} \cap \Lambda_{a}=\Lambda_{A}$, т.е.

$$
\Lambda_{1}=\Lambda_{A} .
$$

Но это значит, что

$$
\left\langle A, B_{1}\right\rangle_{\mathbb{R}} \cap \mathbb{Z}^{n+1}=\left\langle A, B_{1}\right\rangle_{\mathbb{Z}}
$$

т.е. пара векторов $\left\{A, B_{1}\right\}$ дополнима до базиса $\mathbb{Z}^{n+1}$. Лемма 7 доказана.

ЛЕмма 8 (основная). Пусть $\varepsilon>0, \omega, \omega^{\prime} \in S_{f}^{*}, \omega$ освещает мнохество $B_{f}^{1}\left(\omega^{\prime}\right)$ в точке начала координат. Пусть $\Pi \subset \mathbb{R}^{n}-n$-мерный замкнутый параллелепипед с выделенной $(n-1)$-мерной гранью $\Gamma$. Пусть $\exists m_{k} \in \mathbb{Z}^{n}$ :

1) $m_{k}$ освещиает множество $B_{f}^{1}(\omega)$ в точке начала координат;

2) для всех $\alpha \in \Pi \backslash \Gamma m_{k}-k$-e f-OHП $\mathscr{L}_{\alpha}$;

3) для всех $\alpha \in \Gamma \quad\left\{\mathscr{L}_{\alpha}\left(m_{k}\right)\right\}=0$;

4) функиия $\left\{\mathscr{L}_{\alpha}\left(m_{k}\right)\right\}$ непрерывна по $\alpha$ в $\Pi$.

Тогда существует $n$-мерный замкнутый параллелепипед $\Pi^{\prime} \subset$ int $\Pi$ с выделенной $(n-1)$-мерной гранъю $\Gamma^{\prime}$ и $m_{k+1} \in \mathbb{Z}^{n}$ :

1) $\left|\omega-\frac{m_{k+1}}{\left|m_{k+1}\right|}\right| \leqslant \varepsilon\left|m_{k+1}\right|^{-\Delta(n-1)}$;

2) $m_{k+1}$ освещ,ает мнохсество $B_{f}^{1}\left(\omega^{\prime}\right)$ в точке начала координат;

3) для всех $\alpha \in \Pi^{\prime} \backslash \Gamma^{\prime} m_{k+1}-(k+1)$ - e f-OHП $\mathscr{L}_{\alpha}$;

4) для всех $\alpha \in \Gamma^{\prime}\left\{\mathscr{L}_{\alpha}\left(m_{k+1}\right)\right\}=0$;

5) функиия $\left\{\mathscr{L}_{\alpha}\left(m_{k+1}\right)\right\}$ непрерывна по $\alpha$ в $\Pi^{\prime}$.

Для доказательства основной леммы нам понадобится следующее обозначение. Пусть фиксировано $a \in \mathbb{R}^{n}$. Тогда положим для целых $\nu$

$$
\pi_{\nu}(a)=\left\{\alpha \in \mathbb{R}^{n} \mid \mathscr{L}_{\alpha}(a)=\nu\right\} .
$$

Отметим также следующий очевидный факт. 
УТВЕРЖДЕНИЕ. Для любого $\nu \in \mathbb{Z} \pi_{\nu}(a) \perp$, причем расстояние между $\pi_{\nu}(a)$ $u \pi_{\nu+1}(a)$ равно $1 / \sqrt{a_{1}^{2}+\cdots+a_{n}^{2}}$. Кроме того, для всех $\nu \in \mathbb{Z}$ функиия $\left\{\mathscr{L}_{\alpha}(a)\right\}$ непрерывна по а в слое

$$
\left\{\alpha \in \mathbb{R}^{n} \mid \nu \leqslant \mathscr{L}_{\alpha}(a)<\nu+1\right\} .
$$

ДоКАЗАТЕЛЬСТВО основной лЕмМЫ. Поскольку для всех $\alpha \in \Gamma\left\{\mathscr{L}_{\alpha}\left(m_{k}\right)\right\}=0$, существует точка $A \in \mathbb{M}_{n}\left(m_{k}\right)$ такая, что для всех $\alpha \in \Gamma \overline{\mathscr{L}_{\alpha}}(A)=0$. Но функция $\left\{\mathscr{L}_{\alpha}\left(m_{k}\right)\right\}$ непрерьвна по $\alpha$ в $\Pi$ и для всех $\alpha \in \Pi \backslash \Gamma m_{k}-k$-е $f$-ОНП $\mathscr{L}_{\alpha}$. Поэтому точка $A$ примитивна. Применим к точкам $A$ и $\omega$ лемму 7. Получим бесконечно много точек $b \in \mathbb{Z}^{n}$, удовлетворяющих утверждению леммы 7 .

Для всех таких достаточно далеких $b$ выполняются следующие три условия:

1) $\exists \nu \in \mathbb{Z}: \pi_{\nu}(b) \cap \operatorname{int} \Gamma \neq \varnothing$ и $\pi_{\nu+1}(b) \cap \operatorname{int} \Gamma \neq \varnothing$;

2) $m_{k}$ освещает множество $B_{f}^{|b|}(b)$ в точке начала координат;

3) $b$ освещает множество $B_{f}^{\left|\omega^{\prime}\right|}\left(\omega^{\prime}\right)$ в точке начала координат.

Действительно, первьй пункт вьполняется в силу приведенного выше утверждения и того факта, что $\omega \nmid m_{k}$, ибо $\omega \in S_{f}^{*}$. Заметим, отсюда следует, что $b \nmid m_{k}$. Чтобы выполнялись второй и третий пункты, в силу лемм 1 и 2 достаточно, чтобы $b /|b|$ было близко к $\omega$.

В качестве $m_{k+1}$ возьмем любую такую точку $b$. Тогда $\exists B \in \mathbb{M}_{n}\left(m_{k+1}\right)$ :

1) пара векторов $\{A, B\}$ дополнима до базиса $\mathbb{Z}^{n+1}$;

2) сушествует гиперплоскость $\pi \subset \mathbb{R}^{n}$ такая, что $\pi \cap \operatorname{int} \Gamma \neq \varnothing$ и для всех $\alpha \in \pi$ $\overline{\mathscr{L}_{\alpha}}(B)=0$ (в качестве $\pi$ надо брать $\pi_{\nu}(b)$ или $\left.\pi_{\nu+1}(b)\right)$.

Введем следующие обозначения:

$$
K=\left\{a \in \mathbb{Z}^{n} \backslash\left\{0, m_{k+1}\right\}|| a|\leqslant| m_{k+1} \mid\right\}, \quad K^{-}=K \backslash\left\langle m_{k}, m_{k+1}\right\rangle_{\mathbb{Z}}
$$

Если мы добьемся того, что $\left\{\mathscr{L}_{\alpha}\left(m_{k+1}\right)\right\}<\left\{\mathscr{L}_{\alpha}\left(m_{k}\right)\right\} \leqslant\left\{\mathscr{L}_{\alpha}(a)\right\}$ для любого $a \in$ $K$, кроме $m_{k}$ и $m_{k+1}$, то $m_{k+1}$ будет $(k+1)$-м $f$-ОНП $\mathscr{L}_{\alpha}$. Для этого точки решетки $\left\langle m_{k}, m_{k+1}\right\rangle_{\mathbb{Z}}$ придется рассматривать отдельно, поскольку с этими точками возникают определенные трудности.

Для доказательства леммы достаточно показать, что существуют два замкнутых параллелепипеда $\Pi_{1}$ и $\Pi^{\prime}$ такие, что

$$
\Pi \supseteq \Pi_{1} \supseteq \Pi^{\prime}
$$

удовлетворяющие следующим условиям:

1) для всех $\alpha \in \Pi_{1}$ и $a \in K^{-}\left\{\mathscr{L}_{\alpha}\left(m_{k}\right)\right\}<\left\{\mathscr{L}_{\alpha}(a)\right\}$;

2) $\exists \Gamma^{\prime}-(n-1)$-мерная грань $\Pi^{\prime}$ такая, что для всех $\alpha \in \Gamma^{\prime}\left\{\mathscr{L}_{\alpha}\left(m_{k+1}\right)\right\}=0$ и для всех $\alpha \in \Pi^{\prime} \backslash \Gamma^{\prime}$ и $a \in K\left\{\mathscr{L}_{\alpha}\left(m_{k+1}\right)\right\}<\left\{\mathscr{L}_{\alpha}(a)\right\}$;

3) для всех $\alpha \in \Pi^{\prime}$ и $a \in\left\langle m_{k}, m_{k+1}\right\rangle_{\mathbb{Z}} \backslash\left\{0, m_{k}, m_{k+1}\right\}$ таких, что $|a| \leqslant\left|m_{k+1}\right|$, выполнено неравенство $\left\{\mathscr{L}_{\alpha}\left(m_{k}\right)\right\}<\left\{\mathscr{L}_{\alpha}(a)\right\}$, т.е. $m_{k+1}$ является $(k+1)$-м $f$-ОНП $\mathscr{L}_{\alpha}$ для всех $\alpha \in \Pi^{\prime} \backslash \Gamma^{\prime}$

4) $\Pi^{\prime} \subset \operatorname{int} \Pi$;

5) функция $\left\{\mathscr{L}_{\alpha}\left(m_{k+1}\right)\right\}$ непрерьвна по $\alpha$ в $\Pi^{\prime}$. 
Докажем существование $\Pi_{1}$. Положим

$$
\mathscr{P}=\pi \cap \operatorname{int} \Gamma \text {. }
$$

Поскольку $m_{k+1} \nmid m_{k}$, то $\operatorname{dim} \mathscr{P}=n-2$.

Покажем, что если $a \in \mathbb{Z}^{n}$ и $\nu \in \mathbb{Z}$ таковы, что $\operatorname{dim}\left(\pi \cap \Gamma \cap \pi_{\nu}(a)\right)=n-2$, то точка $a$ лежит в решетке $\left\langle m_{k}, m_{k+1}\right\rangle_{\mathbb{Z}}$. Действительно, тогда существует точка $X \in$ $\mathbb{M}_{n}(a)$ такая, что $\overline{\mathscr{L}_{\alpha}}(X)=\overline{\mathscr{L}}_{\alpha}(A)=\overline{\mathscr{L}_{\alpha}}(B)=0$ для всех $\alpha \in \pi \cap \Gamma \cap \pi_{\nu}(a)$. По лемме 4 точки $A, B, X$ линейно зависимы. Но пара $\{A, B\}$ дополнима до базиса $\mathbb{Z}^{n+1}$. Следовательно, точка $X$ лежит в решетке $\langle A, B\rangle_{\mathbb{Z}}$. Но тогда и точка $a=\underline{X}$ лежит в решетке $\left\langle m_{k}, m_{k+1}\right\rangle_{\mathbb{Z}}$.

Получили, что для всех $a \in K^{-}$и $\nu \in \mathbb{Z}$

$$
\operatorname{dim}\left(\pi_{\nu}(a) \cap \mathscr{P}\right) \leqslant n-3 .
$$

Но множество $\left\{\pi_{\nu}(a) \mid \pi_{\nu}(a) \cap \Gamma \neq \varnothing, a \in K^{-}, \nu \in \mathbb{Z}\right\}$ конечно. Следовательно, $\exists \mathscr{P}^{\prime} \subseteq \mathscr{P}$-куб размерности $n-2$ такой, что для всех $a \in K^{-}, \nu \in \mathbb{Z}$

$$
\pi_{\nu}(a) \cap \mathscr{P}^{\prime}=\varnothing,
$$

т.е. для всех $a \in K^{-}, \alpha \in \mathscr{P}^{\prime}$

$$
\left\{\mathscr{L}_{\alpha}(a)\right\} \neq 0 .
$$

Кроме того, поскольку $\mathscr{P}^{\prime} \subseteq \Gamma$, для всех $\alpha \in \mathscr{P}^{\prime}$

$$
\left\{\mathscr{L}_{\alpha}\left(m_{k}\right)\right\}=0 .
$$

Тогда из непрерывности функции $\left\{\mathscr{L}_{\alpha}\left(m_{k}\right)\right\}$ по $\alpha$ в $\Pi$ и того факта, что $\mathscr{P}^{\prime}$ находится во внутренности слоя непрерывности $\left\{\mathscr{L}_{\alpha}(a)\right\}$ для любого $a \in K^{-}$, получаем, что существует замкнутый параллелепипед $\Pi_{1} \subset \Pi$ такой, что $\mathscr{P}^{\prime}$ содержится во внутренности некоторой его $(n-1)$-мерной грани, и такой, что для всех $a \in K^{-}$и $\alpha \in \Pi_{1}$

$$
\left\{\mathscr{L}_{\alpha}\left(m_{k}\right)\right\}<\left\{\mathscr{L}_{\alpha}(a)\right\} .
$$

Теперь докажем существование $\Pi^{\prime}$. Положим

$$
\mathscr{R}=\pi \cap \operatorname{int} \Pi_{1} .
$$

Очевидно, $\operatorname{dim} \mathscr{R}=n-1$.

Если $a \nmid m_{k+1}$, то для всех $\nu \in \mathbb{Z} \pi \neq \pi_{\nu}(a)$. Но $m_{k+1}$ не обязательно примитивна. Допустим, существуют такие $q, \nu \in \mathbb{Z}$, что $m_{k+1} / q \in \mathbb{Z}^{n}$ и $\pi=\pi_{\nu}\left(m_{k+1} / q\right)$. Тогда сушествует целая точка $X \in \mathbb{M}_{n}\left(m_{k+1} / q\right)$ такая, что $\overline{\mathscr{L}}_{\alpha}(X)=0$ для всех $\alpha \in \pi$. Но для всех таких $\alpha \overline{\mathscr{L}}_{\alpha}(B)=0$, т.е. $B=q X$, в то время, как точка $B$ примитивна. Значит, $|q| \leqslant 1$. Стало быть, для всех $a \in K \backslash\left\{-m_{k+1}\right\}$ и $\nu \in \mathbb{Z} \pi \neq \pi_{\nu}(a)$, т.е.

$$
\operatorname{dim}\left(\pi_{\nu}(a) \cap \mathscr{R}\right) \leqslant n-2 .
$$

Следовательно, $\exists \mathscr{R}^{\prime} \subseteq \mathscr{R}$ - куб размерности $n-1$ такой, что для всех $a \in K \backslash\left\{-m_{k+1}\right\}$ и $\nu \in \mathbb{Z}$

$$
\pi_{\nu}(a) \cap \mathscr{R}^{\prime}=\varnothing,
$$


т.е. для всех $a \in K \backslash\left\{-m_{k+1}\right\}, \alpha \in \mathscr{R}^{\prime}$

$$
\left\{\mathscr{L}_{\alpha}(a)\right\} \neq 0 \text {. }
$$

Кроме того, поскольку $\mathscr{R}^{\prime} \subset \pi$, для всех $\alpha \in \mathscr{R}^{\prime}$

$$
\left\{\mathscr{L}_{\alpha}\left( \pm m_{k+1}\right)\right\}=0 .
$$

Пусть $\mathscr{E} \mathscr{R}$ - такой ортонормированньй базис $\mathbb{R}^{n}$ (здесь мы рассматриваем $\mathbb{R}^{n}$ как аффинное пространство, т.е. начало координат можно сдвинуть), в котором $\mathscr{R}^{\prime}$ имеет вид

$$
\mathscr{R}^{\prime}=\left\{\alpha=\left(\alpha_{1}, \ldots, \alpha_{n}\right)\left|\alpha_{1}=0,\right| \alpha_{i} \mid \leqslant \delta_{0}, i=2,3, \ldots, n\right\} .
$$

Тогда из того факта, что $\mathscr{R}^{\prime}$ находится во внутренности слоя непрерьвности $\left\{\mathscr{L}_{\alpha}(a)\right\}$ для любого $a \in K \backslash\left\{-m_{k+1}\right\}$ получаем, что существует положительное $\delta_{1}$ такое, что для всех $a \in K$ и $\alpha \in \Pi^{\prime} \backslash \pi$

$$
\left\{\mathscr{L}_{\alpha}\left(m_{k+1}\right)\right\}<\left\{\mathscr{L}_{\alpha}(a)\right\},
$$

где $\Pi^{\prime}$ в базисе $\mathscr{E} \mathscr{R}$ имеет вид либо

$$
\Pi^{\prime}=\left\{\alpha \in \mathbb{R}^{n}\left|0 \leqslant \alpha_{1} \leqslant \delta_{1},\right| \alpha_{i} \mid \leqslant \delta_{1}, i=2,3, \ldots, n\right\},
$$

либо

$$
\Pi^{\prime}=\left\{\alpha \in \mathbb{R}^{n}\left|-\delta_{1} \leqslant \alpha_{1} \leqslant 0,\right| \alpha_{i} \mid \leqslant \delta_{1}, i=2,3, \ldots, n\right\},
$$

в зависимости от того, с какой стороны от плоскости $\pi$ находится слой непрерывности функции $\left\{\mathscr{L}_{\alpha}\left(m_{k+1}\right)\right\}$. Из-за малости $\delta_{1}$ можно считать, что $\Pi^{\prime} \subset \operatorname{int} \Pi_{1}$.

Положим

$$
\Gamma^{\prime}=\pi \cap \Pi^{\prime}
$$

и заметим, что $\left\{\mathscr{L}_{\alpha}\left(m_{k+1}\right)\right\}=0$ для всех $\alpha \in \Gamma^{\prime}$. Кроме того, функция $\left\{\mathscr{L}_{\alpha}\left(m_{k+1}\right)\right\}$ непрерьвна в $\Pi^{\prime}$. Следовательно, остается показать, что для всех $\alpha \in \Pi^{\prime}$ и $a \in\left\langle m_{k}, m_{k+1}\right\rangle_{\mathbb{Z}} \backslash\left\{0, m_{k}, m_{k+1}\right\}$ таких, что $|a| \leqslant\left|m_{k+1}\right|$, выполнено неравенство $\left\{\mathscr{L}_{\alpha}\left(m_{k}\right)\right\}<\left\{\mathscr{L}_{\alpha}(a)\right\}$, и тогда лемма будет доказана.

Для этого введем следующие обозначения:

$\mathscr{M}=\langle A, B\rangle_{\mathbb{R}} \cap\left\{x \in \mathbb{R}^{n+1}|| \underline{x}|\leqslant| m_{k+1} \mid\right\}, \quad \ell_{\alpha}=\langle A, B\rangle_{\mathbb{R}} \cap\left\{x \in \mathbb{R}^{n+1} \mid \overline{\mathscr{L}_{\alpha}}(x)=0\right\} ;$ $\mathscr{M}$ - вьпуклое, центрально симметричное, замкнутое 2-мерноемножество, точка $B$ принадлежит границе множества $\mathscr{M}$, a $\ell_{\alpha}-$ прямая, проходящая через 0.

Поскольку $\alpha \in \Pi^{\prime}$, то $A$ и $B$ лежат по одну сторону от гиперплоскости

$$
\left\{x \in \mathbb{R}^{n+1} \mid \overline{\mathscr{L}_{\alpha}}(x)=0\right\}
$$

(или может быть на ней), причем $B$ лежит к ней ближе, чем $A$. Следовательно, и в двумерной плоскости $\langle A, B\rangle_{\mathbb{R}}$ точки $A$ и $B$ лежат по одну сторону от прямой $\ell_{\alpha}$, причем $B$ к ней ближе, чем $A$.

Докажем, что среди всех целых точек множества $\mathscr{M}$, лежащих с точкой $A$ по одну сторону от $\ell_{\alpha}$, кроме $B$ и начала координат, точка $A$ ближайшая к прямой $\ell_{\alpha}$. Воспользуемся тем, что $m_{k}$ освещает множество $B_{f}^{\left|m_{k+1}\right|}\left(m_{k+1}\right)$ в точке начала координат. Отсюда следует, что $\left|m_{k}-m_{k+1}\right|>\left|m_{k}\right|$, и, стало быть, $A-B \notin \mathscr{M}$. Но $\pm B \in \partial \mathscr{M}$, $\pm A \in \operatorname{int} \mathscr{M}$, а множество $\mathscr{M}$ вьпукло; следовательно, все точки вида $\pm(B-\lambda A+\mu(A+$ $B)$ ) с натуральньми $\lambda$ и $\mu$ лежат вне $\mathscr{M}$. Поэтому достаточно рассмотреть точки вида $\lambda A+\mu(A+B), \lambda, \mu \in \mathbb{N}$. Но среди них точка $A$ - ближайшая к $\ell_{\alpha}$, поскольку $(\lambda-1) A+\mu(A+B)$ лежат по ту же сторону от $\ell_{\alpha}$, что и $A$. Это значит, что для всех $\alpha \in \Pi^{\prime}$ и $a \in\left\langle m_{k}, m_{k+1}\right\rangle_{\mathbb{Z}} \backslash\left\{0, m_{k}, m_{k+1}\right\}$ таких, что $|a| \leqslant\left|m_{k+1}\right|$, выполнено неравенство $\left\{\mathscr{L}_{\alpha}\left(m_{k}\right)\right\}<\left\{\mathscr{L}_{\alpha}(a)\right\}$.

Лемма доказана. 


\section{7. Доказательство теорем.}

ДоКАЗАТЕЛЬСТВО ТЕОРЕМЫ 3. Проведем индукцию по вложенньм параллелепипедам.

(I) Основание индукции. В качестве $m_{1}$ возьмем точку $m$, о которой говорится в условии теоремы. Для всех $\alpha \in \pi_{0}\left(m_{1}\right)$

$$
\mathscr{L}_{\alpha}\left(m_{1}\right)=0
$$

Ясно, что можно выбрать такое $\alpha^{\prime} \in \pi_{0}\left(m_{1}\right)$, что для всех $a \in \mathbb{Z}^{n} \cap S_{f} \backslash\left\{ \pm m_{1}\right\}$

$$
\mathscr{L}_{\alpha^{\prime}}(a) \neq 0 \text {. }
$$

Это значит, что для любого $a \in \mathbb{Z}^{n} \cap S_{f} \backslash\left\{ \pm m_{1}\right\}$ точка $\alpha^{\prime}$ лежит во внутренности слоя непрерьвности функции $\mathscr{L}_{\alpha}(a)$. Следовательно, существует положительное $\delta$ такое, что, если обозначить через $\mathscr{E}_{\pi}$ базис $\mathbb{R}^{n}$ с началом координат в $\alpha^{\prime}$, в котором плоскость $\pi_{0}\left(m_{1}\right)$ имеет вид $\left\{\alpha \in \mathbb{R}^{n} \mid \alpha_{1}=0\right\}$ и первая координата вектора $m_{1}$ положительна, то для всех $a \in \mathbb{Z}^{n} \cap S_{f} \backslash\left\{m_{1}\right\}$ и $\alpha \in \Pi_{1} \backslash \pi_{0}\left(m_{1}\right)$

$$
\left\{\mathscr{L}_{\alpha}\left(m_{1}\right)\right\}<\left\{\mathscr{L}_{\alpha}(a)\right\},
$$

где $\Pi_{1}$ в базисе $\mathscr{E}_{\pi}$ имеет вид

$$
\Pi_{1}=\left\{\alpha \in \mathbb{R}^{n}\left|0 \leqslant \alpha_{1} \leqslant \delta,\right| \alpha_{i} \mid \leqslant \delta, i=2,3, \ldots, n\right\} .
$$

Положим

$$
\Gamma_{1}=\Pi_{1} \cap \pi_{0}\left(m_{1}\right) .
$$

Тогда для всех $\alpha \in \Pi_{1} \backslash \Gamma_{1} m_{1}$ является первьпм $f$-ОНП $\mathscr{L}_{\alpha}$, в то время как $\mathscr{L}_{\alpha}\left(m_{1}\right)=0$ для всех $\alpha \in \Gamma_{1}$. Легко видеть, что функция $\left\{\mathscr{L}_{\alpha}\left(m_{1}\right)\right\}$ непрерывна по $\alpha$ в $\Pi_{1}$. Наконец, по условию теоремы $m_{1}$ освещает множество $B_{f}^{1}\left(\omega_{2}\right)$ в точке начала координат.

(II) Шаг индукции. Пусть $\Pi_{k}-n$-мерный замкнутьй параллелепипед с выделенной $(n-1)$-мерной гранью $\Gamma_{k}$. Пусть существуют $m_{1}, \ldots, m_{k} \in \mathbb{Z}^{n}$ такие, что

1) $m_{k}$ освещает множество $B_{f}^{1}\left(\omega_{k+1}\right)$ в точке начала координат;

2) для всех $\alpha \in \Pi_{k} \backslash \Gamma_{k} m_{i}-i$-е $f$-ОНП $\mathscr{L}_{\alpha}, i=1, \ldots, k$;

3) для всех $\alpha \in \Gamma_{k}\left\{\mathscr{L}_{\alpha}\left(m_{k}\right)\right\}=0$;

4) функция $\left\{\mathscr{L}_{\alpha}\left(m_{k}\right)\right\}$ непрерывна по $\alpha$ в $\Pi_{k}$.

По условию теоремы $\omega_{k+1}$ освещает множество $B_{f}^{1}\left(\omega_{k+2}\right)$ в точке начала координат. Применив основную лемму, получим, что существует $n$-мерньй замкнутьй параллелепипед $\Pi_{k+1} \subset \operatorname{int} \Pi_{k}$ с выделенной $(n-1)$-мерной гранью $\Gamma_{k+1}$ и $m_{k+1} \in \mathbb{Z}^{n}$ :

1) $\left|\omega_{k+1}-\frac{m_{k+1}}{\left|m_{k+1}\right|}\right| \leqslant \varepsilon_{k+1}\left|m_{k+1}\right|^{-\Delta(n-1)}$;

2) $m_{k+1}$ освещает множество $B_{f}^{1}\left(\omega_{k+2}\right)$ в точке начала координат;

3) для всех $\alpha \in \Pi_{k+1} \backslash \Gamma_{k+1} m_{k+1}-(k+1)$-е $f$-ОНП $\mathscr{L}_{\alpha}$;

4) для всех $\alpha \in \Gamma_{k+1}\left\{\mathscr{L}_{\alpha}\left(m_{k+1}\right)\right\}=0$;

5) функция $\left\{\mathscr{L}_{\alpha}\left(m_{k+1}\right)\right\}$ непрерьвна по $\alpha$ в $\Pi_{k+1}$.

(III) Получаем последовательность замкнутых параллелепипедов $\left\{\Pi_{k}\right\}_{k=1}^{\infty}$, обладающую следующим свойством:

$$
\Pi_{k+1} \subset \operatorname{int} \Pi_{k} \quad \forall k \in \mathbb{N} \text {. }
$$

Следовательно, $\exists \alpha \in \mathbb{R}^{n}: \alpha \in \operatorname{int} \Pi_{k} \forall k \in \mathbb{N}$, для которого и будет выполнено утверждение теоремы. 
ДОКАЗАТЕЛЬСТВО ТЕОРЕМЫ 2. По условию множество $\Omega$ асимптотически допустимо. Пусть $\left\{\theta_{i}\right\}_{i=1}^{\infty}-$ последовательность в $S_{f}$, о которой говорится в определении 5 . Мы можем считать, что $\left\{\theta_{i}\right\}_{i=1}^{\infty} \subset S_{f}^{*}$. Действительно, поскольку $S_{f}^{*}$ всюду плотно в $S_{f}$, то, применяя леммы 1,2 последовательно к точкам $\left\{\theta_{i}\right\}_{i=1}^{\infty}$, мы можем пошевелить их так, чтобы они все стали принадлежать $S_{f}^{*}$, свойство освещаемости сохранилось и множество предельных точек осталось прежним. Не ограничивая общности, можно также считать, что существует целая точка $m \in S_{f}$, освещающая множество $B_{f}^{1}\left(\theta_{2}\right)$ в точке начала координат. Пусть $\sigma \in \Omega,\left\{\theta_{i_{j}}\right\}_{j=1}^{\infty}-$ подпоследовательность, сходящаяся к $\sigma$ (будем считать, что $i_{1}>2$ ). Из лемм 1 и 2 следует, что существует последовательность $\left\{\tau_{j}\right\}_{j=1}^{\infty} \subset S_{f}^{*}$ такая, что $\forall j\left|\theta_{i_{j}}-\tau_{j}\right|=\delta_{j} \neq 0, \delta_{j} \rightarrow 0$, и при этом $\theta_{i_{j}-1}$ освещает множество $B_{f}^{1}\left(\tau_{j}\right)$ в точке начала координат, а $\tau_{j}$ освещает $B_{f}^{1}\left(\theta_{i_{j}+1}\right)$ в точке начала координат.

Определим последовательность $\left\{\omega_{i}\right\}_{i=1}^{\infty}$ следующим образом: $\omega_{i_{j}}$ положим равным либо $\theta_{i_{j}}$, либо $\tau_{j}$; если же $i \neq i_{j}$, то положим $\omega_{i}=\theta_{i}$. Заметим, что таких последовательностей континуум.

Определим последовательность $\left\{\varepsilon_{i}\right\}_{i=1}^{\infty} \subset \mathbb{R}_{+}$так, чтобы она убьвала и чтобы выполнялось $\varepsilon_{i_{j}}<\delta_{j} / 2$ и $\left|\omega_{1}-m\right| \leqslant \varepsilon_{1}$.

Применим к последовательностям $\left\{\omega_{i}\right\}_{i=1}^{\infty}$ и $\left\{\varepsilon_{i}\right\}_{i=1}^{\infty}$ теорему 3 . Получим континуум требуемых линейных форм. Все эти формы будут попарно различны, поскольку для любых двух различных последовательностей $\left\{\omega_{i}\right\}_{i=1}^{\infty}$ и $\left\{\omega_{i}^{\prime}\right\}_{i=1}^{\infty}$ существует такое $i$, что $\left|\omega_{i}-\omega_{i}^{\prime}\right|>2 \varepsilon_{i}$, и, стало быть, соответствующие $i$-е $f$-ОНП различны.

Теорема доказана.

В заключение автор хотел бы выразить искреннюю благодарность Н. Г. Мощевитину за неоценимую помощ в работе над статьей и многочисленные обсуждения результатов.

\section{СПИСОК ЦИТИРОВАННОЙ ЛИТЕРАТУРЫ}

[1] Rogers C. A. The asymptotic directions of $n$ linear forms in $n+1$ integral variables // Proc. London Math. Soc. Ser. 2. 1951. V. 52. P. 161-185.

[2] Rogers C. A. The signatures of the errors of simultaneous Diophantine approximations // Proc. London Math. Soc. Ser. 2. 1951. V. 52. P. 186-190.

[3] Мощевитин Н. Г. Наилучшие совместные приближения: нормы, сигнатуры и асимптотические направления // Матем. заметки. 2000. Т. 67. № 5. С. 730-737.

[4] Финкельштейн Ю. Ю. Полигоны Клейна и приведенные регулярные непрерьвные дроби // УМН. 1993. Т. 48. № 3. С. 205-206.

[5] Болтянский В. Г., Гохберг И. Ц. Теоремы и задачи комбинаторной геометрии. М.: Наука, 1965.

[6] Мощевитин Н. Г. О наилучших совместных приближениях // УМН. 1996. Т. 51. №6. C. 213-214.

[7] Glyn Harman. On the distribution of $\alpha p$ modulo one. II // Proc. London Math. Soc. Ser. 3. 1996. V. 72. P. 241-260.

[8] Glyn Harman. Simultaneous Diophantine approximations with primes // J. London Math. Soc. Ser. 2. 1989. V. 39. P. 405-413.

[9] Sos V. T., Szekeres G. Rational approximation vectors // Acta Arith. 1988. V. 49. №3. P. 255-261.

[10] Виноградов И. М. Особые варианты метода тригонометрических сумм. М.: Наука, 1976. 